\title{
Estudo do Pré-tratamento e da Hidrólise do Bagaço de Cana Utilizando a Vermiculita Ativada com Ácido como Catalisador
}

\author{
B. R. P. NUNES ${ }^{1}$, L. de S.CONRADO ${ }^{2}$ e C. R. da S. MORAIS ${ }^{3}$ \\ ${ }^{1}$ Universidade Federal de Campina Grande, Programa de Pós-Graduação em Engenharia Química \\ ${ }^{2}$ Universidade Federal de Campina Grande, Unidade Acadêmica de Engenharia Química \\ ${ }^{3}$ Universidade Federal de Campina Grande, Unidade Acadêmica de Engenharia de Materiais \\ E-mail para contato: brunimeq@gmail.com
}

\begin{abstract}
RESUMO - Visando a obtenção de glicose estudaram-se os pré-tratamentos hidrotérmico e químico $\left(\mathrm{NaOH}+\mathrm{H}_{2} \mathrm{SO}_{4}\right)$ e a hidrólise do bagaço de cana-de-açúcar utilizando como catalisador a argila vermiculita tratada com ácido. Amostras do bagaço, antes e após os tratamentos, foram caracterizadas quanto ao teor de fibras. Verificou-se que o prétratamento químico apresentou resultados mais satisfatórios o qual proporcionou uma diminuição de $40 \%$ no teor de lignina e de $43 \%$ no teor de hemicelulose, com relação ao bagaço in natura. Testes catalíticos em solução aquosa foram conduzidos a $200{ }^{\circ} \mathrm{C}$, visando avaliar a utilização da vermiculita tratada como catalisador da hidrólise do bagaço de cana. O produto reacional foi filtrado e o sobrenadante foi analisado por cromatografia líquida de alta eficiência. Alcançaram-se rendimentos de 13,51\% em glicose e de 65,48 $\%$ em xilose.
\end{abstract}

\section{INTRODUÇÃO}

As mudanças climáticas e a elevação nos custos do petróleo em conjunto com as estratégias para produção de energia têm motivado a busca pelo desenvolvimento de combustíveis alternativos, preferencialmente de fontes renováveis (Buckeridge et al., 2011). A celulose é um dos mais abundantes recursos renováveis em todo o mundo. Devido às vantagens de absorção de carbono na fotossíntese este material pode desempenhar um papel importante como recurso alternativo na produção de bioprodutos e na substituição de combustíveis fósseis (Chen et al., 2011).

A produção de bioprodutos, destacando-se o bioetanol, pode ser realizada através da utilização de diversas matérias-primas lignocelulósicas, como por exemplo, o bagaço de cana-de-açúcar. O bagaço de cana apresenta como vantagens a produção em grande quantidade, o fato de se apresentar processado das moendas e de se encontrar pronto para uso no local (Aguiar, 2010).

Para que o material lignocelulósico seja utilizado como matéria-prima fermentescível, faz-se necessária a aplicação de um pré-tratamento, visando à eliminação, ou diminuição, da lignina e da hemicelulose, bem como a desestruturação da fase cristalina da celulose e o aumento da porosidade do material, de maneira a torná-lo susceptível a hidrólise. Os processos empregados podem ser físicos, químicos, biológicos ou combinados (Dias, 2011). 
A hidrólise da celulose presente na biomassa é o principal processo para sua utilização na produção de bioetanol. Este processo pode ser realizado por meio da hidrólise com ácidos minerais, enzimas, ou água comprimida a alta pressão. Recentemente, a utilização de catalisadores sólidos para a conversão de celulose, tais como, óxidos metálicos de metais de transição, heteropoliácidos, zeólitas, argilas e outros, tem sido investigada. Os catalisadores sólidos apresentam como vantagens a possibilidade de separação do catalisador do produto, a capacidade de ser reutilizado, o baixo nível de desgaste dos equipamentos industriais, a menor geração de efluentes (Silva et al., 2012) além de apresentar elementos considerados importantes para a modificação da sua superfície como a elevada área superficial, presença de grande quantidade de grupos hidroxila e a alta estabilidade (Ogaki et al., 2011). Com isso, realizou-se o estudo da aplicação da argila vermiculita tratada com ácido como catalisador do processo de hidrólise do bagaço de cana-de-açúcar pré-tratado.

\section{MATERIAIS E MÉTODOS}

\subsection{Ativação Ácida da Argila}

A ativação ácida foi realizada partindo-se de uma amostra de argila vermiculita, na granulometria 115 Mesh, Figura 1(a), cedida por pesquisadores do Laboratório de Armazenagem do Programa de Pós-Graduação em Ciências e Engenharia de Materiais da Universidade Federal de Campina Grande, sendo sua composição química em maior parte $\mathrm{SiO}_{2}(40,31 \%), \mathrm{Al}_{2} \mathrm{O}_{3}(14,16 \%)$ e $\mathrm{MgO}(23,48 \%)$, com diâmetro médio das partículas de $22,75 \mu \mathrm{m}$ e capacidade de troca catiônica (CTC) de 115,0 meq/100g (Silva, 2012).

A metodologia utilizada baseou-se nos procedimentos utilizados por Alves (2009), na ativação ácida da vermiculita. Adicionou-se $10 \mathrm{~mL}$ de solução de ácido por grama da argila, mantida sob agitação a $95{ }^{\circ} \mathrm{C}$ durante 4,0 h, conforme o exposto na Figura 2. Os ácidos utilizados foram o sulfúrico $\left(\mathrm{H}_{2} \mathrm{SO}_{4}\right)$ a $3 \mathrm{~mol} / \mathrm{L}$ e o fosfórico $\left(\mathrm{H}_{3} \mathrm{PO}_{4}\right)$ a $4 \mathrm{~mol} / \mathrm{L}$. Em seguida a mistura foi decantada e o sólido obtido foi filtrado à vácuo, lavado exaustivamente com água destilada até $\mathrm{pH}$ neutro e seco em estufa por $24 \mathrm{~h}$ a $105{ }^{\circ} \mathrm{C}$, Figura 1(b).

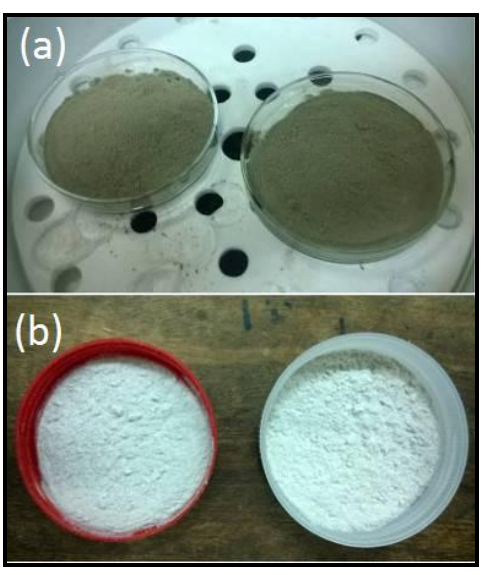

Figura 1 - Argila vermiculita in natura (a) e tratada com ácido (b).

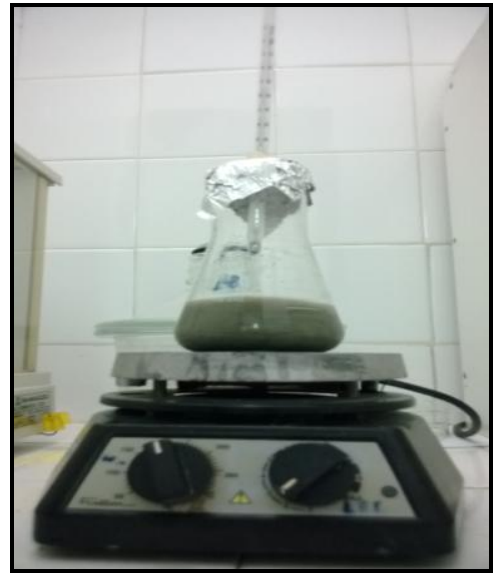

Figura 2 - Tratamento ácido da argila vermiculita. 


\subsection{Caracterização do Bagaço de Cana de Açúcar}

O bagaço de cana-de-açúcar in natura foi submetido à moagem em um moinho de facas, do modelo MA048 da Marconi, para redução de tamanho resultando uma granulometria de $30 \mathrm{Mesh}$. As determinações dos teores de umidade, cinzas, extrativos, holocelulose, celulose e lignina do bagaço de cana antes e após os pré-tratamentos foram realizadas de acordo com os procedimentos para análise lignocelulósica utilizados por Morais et al. (2010).

\subsection{Pré-Tratamento do bagaço de Cana de Açúcar}

O estudo dos pré-tratamentos, hidrotérmico e químico, do bagaço de cana foi realizado em um forno MAITEC com um reator de aço inox de alta pressão, com capacidade de $750 \mathrm{~mL}$, com sistema de controle de temperatura interna e externa, mostrado na Figura 3.

Para o tratamento hidrotérmico utilizou-se uma razão de massa de bagaço/volume de água destilada de $1 / 10$. O tempo de reação foi de $20 \mathrm{~min}$ a uma temperatura de $160{ }^{\circ} \mathrm{C}$. Para o tratamento químico utilizou-se o bagaço in natura sem umidade e uma solução de hidróxido de sódio (NaOH) 4 \% na razão de massa/volume de $1 / 10$. O tempo de reação foi de $60 \mathrm{~min}$ e a temperatura de $120{ }^{\circ} \mathrm{C}$. $\mathrm{O}$ material obtido foi lavado até $\mathrm{pH}$ neutro, seco em estufa à $105^{\circ} \mathrm{C}$, pesado e transferido para o reator onde se adicionou uma solução de ácido sulfúrico $\left(\mathrm{H}_{2} \mathrm{SO}_{4}\right) 3 \%$ na razão de massa/volume de $1 / 10$. O tempo de reação foi de 60 min e a temperatura de $120{ }^{\circ} \mathrm{C}$. $\mathrm{O}$ material resultante foi lavado até $\mathrm{pH}$ neutro e seco em estufa até massa constante.

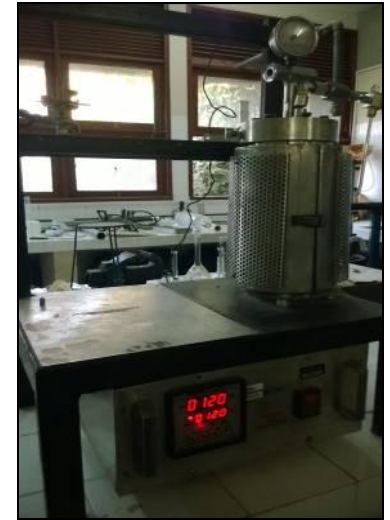

Figura 3 - Reator utilizado no pré-tratamento do bagaço.

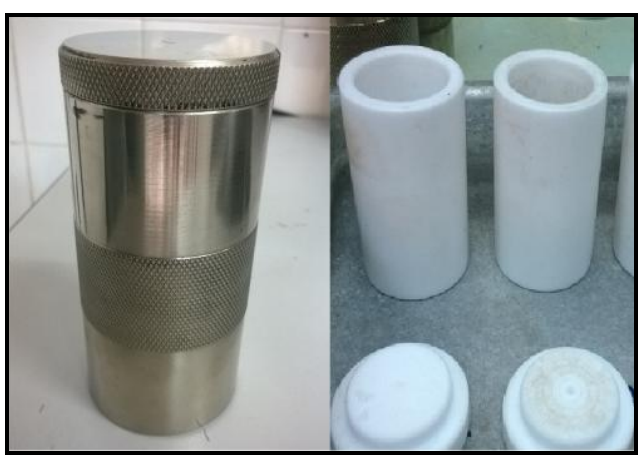

Figura 4 - Autoclaves utilizadas na realização dos testes catalíticos.

\subsection{Testes Catalíticos}

Os testes catalíticos foram conduzidos em autoclaves de politetrafluoretileno revestidas por peças de aço inox, Figura 4, sendo as reações conduzidas em estufa a $200{ }^{\circ} \mathrm{C}$, por períodos de tempo de 1, 2 e 3 horas. Para cada reação utilizou-se 0,25 g de bagaço de cana pré-tratado (BPT), $20 \mathrm{~g}$ de água destilada e $0,5 \mathrm{~g}$ de argila vermiculita ativada. 
Foram realizados ensaios sem a presença do catalisador com o objetivo de visualizar a quantidade de açúcares obtida e em seguida, por meio de comparação, verificar se os resultados para a reação na presença da argila foram significativos.

As condições utilizadas durante a realização dos testes se encontram na Tabela 1. Os ensaios foram realizados utilizando amostras do bagaço pré-tratado (BPT) pelos dois métodos em estudo. As argilas tratadas com ácido, utilizadas como catalisador, foram nomeadas como argila $\left(\mathrm{H}_{2} \mathrm{SO}_{4}\right)$, para argila tratada com ácido sulfúrico, e argila $\left(\mathrm{H}_{3} \mathrm{PO}_{4}\right)$, para a argila tratada com ácido fosfórico.

Tabela 1 - Condições utilizadas durante a realização dos testes catalíticos

\begin{tabular}{|c|c|c|c|c|c|c|}
\hline Ensaios & Biomassa & Catalisador & $\begin{array}{c}\text { Tempo de } \\
\text { Reação }(\mathbf{h})\end{array}$ & $\begin{array}{c}\text { Massa de } \\
\text { bagaço }(\mathbf{g})\end{array}$ & $\begin{array}{c}\text { Massa de } \\
\text { catalisador }(\mathbf{g})\end{array}$ & $\begin{array}{c}\text { Volume de } \\
\text { água }(\mathbf{m L})\end{array}$ \\
\hline 1 & BPT & $* * * *$ & 1 & 0,25 & 0,0 & 20,0 \\
\hline 2 & BPT & Argila $\left(\mathrm{H}_{2} \mathrm{SO}_{4}\right)$ & 1 & 0,25 & 0,5 & 20,0 \\
\hline 3 & BPT & Argila $\left(\mathrm{H}_{3} \mathrm{PO}_{4}\right)$ & 1 & 0,25 & 0,5 & 20,0 \\
\hline 4 & BPT & Argila $\left(\mathrm{H}_{2} \mathrm{SO}_{4}\right)$ & 2 & 0,25 & 0,5 & 20,0 \\
\hline 5 & BPT & Argila $\left(\mathrm{H}_{3} \mathrm{PO}_{4}\right)$ & 2 & 0,25 & 0,5 & 20,0 \\
\hline 6 & BPT & Argila $\left(\mathrm{H}_{2} \mathrm{SO}_{4}\right)$ & 3 & 0,25 & 0,5 & 20,0 \\
\hline 7 & BPT & Argila $\left(\mathrm{H}_{3} \mathrm{PO}_{4}\right)$ & 3 & 0,25 & 0,5 & 20,0 \\
\hline
\end{tabular}

O produto obtido ao término dos testes catalíticos foi filtrado e o sobrenadante foi analisado quanto ao teor de açúcares (glicose, xilose, arabinose) e inibidores (ácido acético, furfural e HMF) (mg/L) por cromatografia líquida de alta eficiência (CLAE). A coluna analítica de aço inox utilizada foi a Hi-Plex H (300 x 7,7 mm) com vazão da fase móvel, solução de ácido sulfúrico a 0,005 M, de $0,6 \mathrm{~mL} / \mathrm{min}$; o detector utilizado foi o de índice de refração. Foram calculados os rendimentos em glicose e xilose, conforme as equações 1 e 2 (Li et al., 2013).

$$
r_{\text {glicose }}(\%)=\frac{0,9 \cdot \mathrm{V} \cdot \mathrm{C}}{W} \text { (1) } \quad r_{\text {xilose }}(\%)=\frac{0,88 \cdot \mathrm{V} \cdot \mathrm{C}}{W}
$$

Onde $\mathrm{V}$ = volume no meio reacional, $\mathrm{C}=$ concentração de glicose ou xilose após a hidrólise e $\mathrm{W}=$ massa de celulose ou hemicelulose presentes no material antes da hidrólise.

\section{RESULTADOS E DISCUSSÃO}

\subsection{Caracterização do Bagaço de Cana-de-Açúcar}

O bagaço de cana utilizado neste estudo foi caracterizado antes e após a aplicação da etapa de pré-tratamento. Os dados relativos à quantidade de extrativos, cinzas, celulose, hemicelulose e lignina insolúvel presentes nas amostras se encontram na Tabela 2. O bagaço de cana in natura apresentou um teor de umidade de 7,68 $\pm 0,16 \%$ enquanto que o bagaço pré-tratado pelo método químico (BPTQ) 3,52 $\pm 0,14 \%$ e pelo método hidrotérmico (BPTH) 6,56 $\pm 018 \%$. 
Tabela 2 - Composição das amostras em valores percentuais (\%)

\begin{tabular}{|c|c|c|c|c|c|}
\hline Bagaço & Cinzas & Extrativos & Celulose & Hemicelulose & Lignina \\
\hline In natura & $0,89 \pm 0,12$ & $6,09 \pm 0,17$ & $36,43 \pm 0,69$ & $34,04 \pm 1,10$ & $14,92 \pm 0,48$ \\
\hline BPTQ & $0,17 \pm 0,01$ & $3,82 \pm 0,30$ & $65,93 \pm 0,69$ & $19,15 \pm 1,38$ & $8,95 \pm 0,38$ \\
\hline BPTH & $0,70 \pm 0,15$ & $1,84 \pm 0,09$ & $39,99 \pm 0,20$ & $38,45 \pm 0,88$ & $17,33 \pm 0,80$ \\
\hline Rabelo (2010) & $3,30 \pm 0,10$ & $2,3 \pm 0,10$ & $34,9 \pm 0,9$ & $22,7 \pm 0,3$ & $29,3 \pm 1,60$ \\
\hline Nascimento (2011) & $4,90 \pm 1,10$ & $5,10 \pm 0,40$ & $38,80 \pm 1,40$ & $29,40 \pm 1,70$ & $21,70 \pm 0,30$ \\
\hline Pietrobon (2008) & $* * * *$ & $* * * *$ & 54,55 & 26,75 & 10,44 \\
\hline $\begin{array}{c}\text { Morais e Broetto } \\
(2012)\end{array}$ & $* * * *$ & $* * * *$ & 47,79 & 35,76 & 13,22 \\
\hline
\end{tabular}

De acordo com os resultados dispostos na Tabela 2 verificou-se que o bagaço de cana in natura apresentou uma composição condizente com as encontradas por outros autores, sendo observadas algumas variações que podem ocorrer devido a fatores como a variedade da espécie, o clima, o método e a época do plantio e da colheita bem como, os diferentes métodos usados para a determinação.

Analisando os dados referentes ao bagaço pré-tratado pode-se observar que o método químico proporcionou uma redução de $40 \%$ no teor de lignina e de $43 \%$ no teor de hemicelulose. Para diferentes variedades do bagaço de cana, Philippini (2012) realizou a etapa de pré-tratamento utilizando a concentração do ácido a $2 \%$, com temperatura de $150{ }^{\circ} \mathrm{C}$ e tempo de residência de 30 min, e em seguida hidróxido de sódio a $1 \%$, com temperatura de $100{ }^{\circ} \mathrm{C}$ e mesmo tempo e obteve uma redução média de 80 e $57 \%$ nos teores de hemicelulose e lignina, respectivamente. Em estudos utilizando ácido sulfúrico com concentrações de $0,5 \%$ a $10 \%$, temperatura de $121{ }^{\circ} \mathrm{C}$ e tempos de reação de 15 a $180 \mathrm{~min}$, seguido do tratamento com hidróxido de sódio nas concentrações de $1 \%$ e 4 $\%$, temperaturas de 100 e $120{ }^{\circ} \mathrm{C}$ e tempos de 30 e $60 \mathrm{~min}$, Rueda (2010) alcançou uma remoção de quase $100 \%$ das frações de hemicelulose e lignina. Com isso, constata-se que a utilização do ácido sulfúrico e do hidróxido de sódio auxilia na remoção da hemicelulose e tem papel importante na deslignificação do bagaço de cana.

A utilização do pré-tratamento hidrotérmico manteve praticamente constante a composição do bagaço, sendo observada uma redução apenas no teor de extrativos. Oliveira (2012) obteve mais de 90 $\%$ de remoção da hemicelulose, no estudo do pré-tratamento hidrotérmico da palha de cana, que apresenta composição semelhante a do bagaço, nas temperaturas de 180 a $195{ }^{\circ} \mathrm{C}$, por períodos de 10 e 15 min. $O$ autor observou que condições mais severas produziram uma maior degradação da hemicelulose, sendo esta iniciada, preferencialmente, a partir dos $180{ }^{\circ} \mathrm{C}$. A pequena alteração na composição do bagaço de cana em estudo pode ser explicada pelo fato da temperatura utilizada no pré-tratamento hidrotérmico ter sido de $160{ }^{\circ} \mathrm{C}$, com isso não permitindo um maior efeito desta variável na ruptura dos grupos acetil presentes na hemicelulose e, por conseguinte a autohidrólise das ligações glicosídicas dos carboidratos (Oliveira, 2012). 


\subsection{Testes Catalíticos}

Após a etapa de Pré-tratamento o bagaço foi submetido a reações na presença do catalisador por períodos de 1,2 e $3 \mathrm{~h}$ na temperatura de $200^{\circ} \mathrm{C}$. Em estudos anteriores as reações foram conduzidas nas temperaturas de 160,180 e $200{ }^{\circ} \mathrm{C}$ e foi possível observar que a temperatura influencia, até certo ponto, de forma diretamente proporcional na reação de hidrólise, por isso, trabalhou-se com a temperatura mais elevada. Os valores médios de rendimento em glicose e xilose obtidos após os testes se encontram na Figura 5.

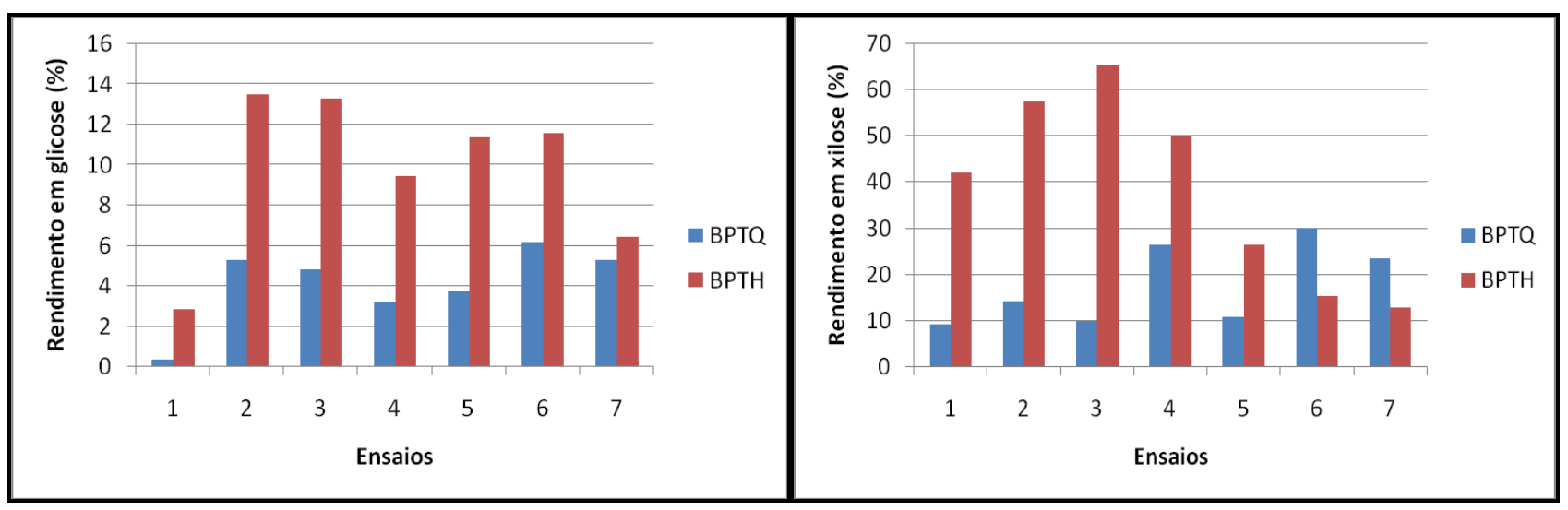

Figura 5 - Rendimentos médios em glicose (a) e xilose (b), obtidos durante os testes catalíticos.

Observando os resultados obtidos após os testes catalíticos nota-se que a utilização das argilas ativadas, comparando com as reações sem a sua presença, proporcionou um aumento nos rendimentos em glicose e xilose, tanto para o BPTQ quanto para o BPTH. Verifica-se que para o BPTH com o aumento do tempo ocorreu uma diminuição nos rendimentos, sendo mais acentuada para o rendimento em xilose, onde os rendimentos caíram de 65 para $15 \%$, utilizando a argila $\left(\mathrm{H}_{3} \mathrm{PO}_{4}\right)$ e de 57 para $12 \%$ utilizando a argila $\left(\mathrm{H}_{2} \mathrm{SO}_{4}\right)$. Essa redução pode ter ocorrido por conta do maior intervalo de tempo que este monossacarídeo ficou exposto à tempertaura de $200{ }^{\circ} \mathrm{C}$ causando assim a sua degradação. Este fato pode ser justificado com a análise do teor de inibidores obtidos no produto reacional, do BPTH, para o tempo de 3 horas, que foi em média de 2,5 g/l, enquanto que para uma hora o máximo foi de $1 \mathrm{~g} / \mathrm{l}$. Analisando os rendimentos em glicose obtidos para o BPTQ, nota-se que os resultados encontram-se na faixa de 3 a $6 \%$ sendo o maior obtido no tempo de $3 \mathrm{~h}$. Na produção de xilose o rendimento sofreu um maior aumento para tempos de exposição maiores, chegando a $30 \%$ em 3 h de reação, utilizando-se a argila $\left(\mathrm{H}_{2} \mathrm{SO}_{4}\right)$.

Os rendimentos obtidos utilizando a argila vermiculita ativada como catalisador da hidrólise do bagaço de cana pré-tratado se encontram de acordo com os resultados obtidos por Tong et al. (2012) que utilizaram a argila montmorilonita ativada com ácido fosfórico na hidrólise da celulose microcristalina, a $200{ }^{\circ} \mathrm{C}$ por $4 \mathrm{~h}$, e obtiveram um rendimento máximo em açúcares de $16,9 \%$. Fang et al. (2011) realizaram o estudo da hidrólise da celulose, a $150{ }^{\circ} \mathrm{C}$ por $24 \mathrm{~h}$, catalisada por hidrotalcita ativada e obtiveram rendimento em glicose, na faixa de 13 a $19 \%$ e conseguiram um aumento para $30,8 \%$ de rendimento quando a massa de catalisador utilizada foi de $2,0 \mathrm{~g}$ para $0,45 \mathrm{~g}$ de celulose microcristalina. 


\section{CONCLUSÕES}

Avaliando os resultados obtidos verificou-se que a aplicação do pré-tratamento químico proporcionou a diminuição dos teores de hemicelulose e lignina, em 40 e $43 \%$, respectivamente, além de manter sem alterações a fração celulose, presentes no bagaço in natura. A utilização do prétratamento hidrotérmico manteve a composição do bagaço, entretanto, será testado em condições mais severas de temperatura visando uma maior degradação da hemicelulose presente no bagaço.

A utilização da argila vermiculita tratada com ácido como catalisador na hidrólise do bagaço de cana provocou um aumento no rendimento em glicose e xilose, comparando-se com a reação sem a sua presença. Os valores máximos obtidos foram 13,51 e $65,48 \%$, em xilose e glicose, respectivamente, para a hidrólise do bagaço pré-tratado pelo método hidrotérmico e 6,18 e 30,07\% em xilose e glicose, respectivamente, para o bagaço pré-tratado pelo método químico.

\section{REFERÊNCIAS}

AGUIAR, C. M. de; Hidrólise Enzimática de Resíduos Lignocelulósicos Utilizando Celulases Produzidas pelo Fungo Aspergillus Níger. Dissertação de Mestrado, Universidade Estadual do Oeste do Paraná, Toledo - PR, 2010.

ALVES, A. P. de M.; Vermiculitas tratadas quimicamente na obtenção de sólidos microporosos como precursores para híbridos inorgânico-orgânicos com aplicações adsortivas. Tese de Doutorado, Universidade Federal da Paraíba, João Pessoa - PB, 2009.

BUCKERIDGE, M. S.; SANTOS, W. D. dos; SOUZA, A. P. de; As Rotas para o Etanol Celulósico no Brasil. Bioetanol de Cana-de-açúcar P\&D para Produtividade e Sustentabilidade, p. 365-380, Editora Blucher, São Paulo, 2010.

CHEN, Y.; LI, G.; YANG, F.; ZHANG, S. M.; Mn/ZSM-5 participation in the degradation of cellulose under phosphoric acid media. Polymer Degradation and Stability, v. 96, p. 863-869, 2011.

DIAS, M. O. de S.; Desenvolvimento e otimização de processos de produção de etanol de primeira e segunda geração e eletricidade a partir da cana-de-açúcar. Tese de Doutorado, Universidade Estadual de Campinas, Campinas - SP, 2011.

FANG, Z.; ZHANG, F.; ZENG, H. Y.; GUO; Production of glucose by hydrolysis of cellulose at $423 \mathrm{~K}$ in the presence of activated hydrotalcite nanoparticles. Bioresource Technology, v. 102, p. 8017-8021, 2011.

LI, S.; GU, Z.; BJORNSON, B. E.; MUTHUKUMARAPPAN, A.; Biochar based solid acid catalyst hydrolyze biomass. Journal of Environmental Chemical Engineering, v. 1, p. 11741181, 2013. 
MORAIS, J. P. S.; ROSA, M. de F.; MARCONCINI, J. M.; Procedimentos para Análise Lignocelulósica, Embrapa - Documentos - 236, Embrapa algodão, Campina Grande - PB, 2010.

MORAIS, A. P. da S.; BROETTO, F.; Pré-Hidrólise Ácida de Bagaço de Cana-de-Açúcar e sua Caracterização Físico-Química. Revista Energia na Agricultura, v.27, p. 1-12, 2012.

NASCIMENTO, V. M.; Pré-tratamento Alcalino (NaOH) do Bagaço de Cana-de-açúcar para a Produção de Etanol e Obtenção de Xilooligômeros. Dissertação de Mestrado, Universidade Federal de São Carlos, São Carlos - SP, 2011.

OGAKI, Y.; SHINOZUKA, Y.; TAKAYOSHI, H.; NOBUYUKI, I.; SHOGO, S.; Hemicellulose decomposition and saccharides production from various plant biomass by sulfonated allophane catalyst. Catalysis Today, v. 164, p. 415-418, 2011.

OLIVEIRA, L. R. M.; Estudo de Alternativas de Pré-tratamento e Hidrólise do Bagaço e Palha de Cana-de-açúcar para Obtenção de Etanol a partir de Celulose. Tese de Doutorado, Universidade de São Paulo, Lorena - SP, 2012.

PHILIPPINI, R. R.; Variedades Híbridas de Bagaço de Cana-de-açúcar: Caracterização Química e Hidrólise Enzimática em Condições de Pré-tratamento Diferenciadas. Dissertação de Mestrado, Universidade de São Paulo, Lorena - SP, 2012.

PIETROBON, V. C.; Hidrólise do Bagaço de Cana-de-açúcar Pré-tratado com Ácido e Álcali Utilizando Enzimas Microbianas Comerciais. Dissertação de Mestrado, Universidade de São Paulo, Piracicaba - SP, 2008.

RABELO, S. C.; Avaliação e Otimização de Pré-tratamentos e Hidrólise Enzimática do Bagaço de Cana-de-açúcar para a Produção de Etanol de Segunda Geração. Tese de Doutorado, Universidade Estadual de Campinas, Campinas - SP, 2010.

RUEDA, S. M. G.; Pré-tratamento e Hidrólise Enzimática do Bagaço de Cana-de-açúcar. Dissertação de Mestrado, Universidade Estadual de Campinas, Campinas - SP, 2010.

SILVA, A. L. Obtenção de Organovermiculita Visando sua Aplicação na Adsorção de Contaminantes Orgânicos. Tese de Doutorado, Universidade Federal de Campina Grande, Campina Grande - PB, 2012.

SILVA, A. S.; Silva, F. L. H da; Carvalho, M. W. N. C.; Pereira, K. R. de O. Hidrólise de Celulose por Catalisadores Mesoestruturados NiO-MCM-41 E MoO3-MCM-41, Química Nova, Vol. 35, nº 4, p. 683-688, 2012.

TONG, D. S.; XIA, X.; LUO, X. P.; WU, L. M.; LIN, C. X.; YU, W. H.; ZHOU, C. H.; ZHONG, Z. K.; Catalytic hydrolysis of cellulose to reducing sugar over acid-activated montmorillonite catalysts. Applied Clay Science, v. 74, p. 147-153, 2012. 